\title{
Biology of BMP signalling and cancer
}

\author{
Moisés Blanco Calvo ${ }^{1}$, Victoria Bolós Fernández ${ }^{2}$, Vanessa Medina Villaamil ${ }^{1,3}$, \\ Guadalupe Aparicio Gallego ${ }^{3}$, Silvia Díaz Prado ${ }^{1,4}$, Enrique Grande Pulido ${ }^{2}$ \\ 1. Institute for Biomedical Research of A Coruña (INIBIC), A Coruña University Hospital, A Coruña, Spain \\ 2. Oncology Medical Department, Pfizer Spain, Alcobendas, Madrid, Spain \\ 3. Oncology Research Unit, A Coruña University Hospital, A Coruña, Spain \\ 4. Medicine Department, University of A Coruña, A Coruña, Spain
}

\begin{abstract}
In recent years, it has been proposed that tumours are not homogeneous but composed of several cellular types like normal tissues. A cellular subtype, which is though to be the origin of tumours as well as their malignant properties (i.e., capacity for regrowth and metastasis), are the cancer stem cells (CSCs). CSCs, like normal stem cells, have a nearly unlimited capacity to self-renew and to proliferate so that are responsible, besides their same auto-perpetuation giving rise to the features previously depicted, also for the generation of the bulk of more differentiated cells in tumour. The altered behaviour of CSCs may be caused by the malfunction of a number of signalling pathways involved in normal embryonic development and in tissue homeostasis in adulthood. Among these signalling pathways are Wnt, Hedgehog, Notch and BMP pathways. In this review, we will focus on the study of molecular aspects of BMP signalling as well as its involvement in cancer.
\end{abstract}

Keywords

BMP pathway; Signalling; Cancer

\section{Introduction}

Bone morphogenetic proteins (BMPs) are a protein family secreted to the extracellular environment as an intercellular communication mechanism and whose mission is work as ligands of specific receptors that are on target cells. BMPs are a subfamily of ligands belonging to the transforming growth factor- $\beta$ (TGF- $\beta$ ) superfamily, which owes its name to the protein that opened the list of ligands of one of the most complex pathways uncovered so far. Despite the large amount of ligands, receptors and other molecules involved in the multiple processes in which the BMP/TGF- $ß$ pathway plays some role, this pathway displays a high homogeneity and functional coherence. Thus, all ligands bind to two types of receptors (type I and type II), which are two trans-membrane serine/threonine kinases. These receptors, in the absence of a signal, are separated in the plasma membrane, but when the ligand is in the environment both receptors are associated, working the molecule ligand as a bridge be-tween the two receptors. In type II receptor, serine/threonine kinase activity is constitutive, so that when it is associated with type I receptor, this is phosphorylated. Thus, the type I receptor is activated and is capable of binding a number of intracellular proteins, which are responsible for transducing the signal to the nucleus. These proteins belong to the Smad protein family, which owes its name to the homology with Sma proteins from the nematode Caenorhabditis elegans and Mad (mothers against decapentaplegic) proteins from the fly Drosophila melanogaster. There are three groups of Smad proteins that are named by putting a letter in front ac-cording to the role they play. Thus, R-Smads (receptor- 
regulated Smads) are Smads that interact with receptors. The Co-Smad (only Smad4) is so called because it is the com-mon mediator of the whole intricate network of signalling derived from ligands of the TGF- $B$ superfamily. Finally, I-Smads (inhibitory Smads) are Smads that inhibit RSmads' activation or their interaction with Co-Smads. When the R-Smads bind to activated type I receptor, they are phosphory-lated and released in their active form in the cytoplasm. The activated $\mathrm{R}$-Smad binds to Co-Smad, forming a complex that translocates to the nucleus. This complex, together with a number of transcription factors, is capable of driving the expression of target genes (Fig. 1).

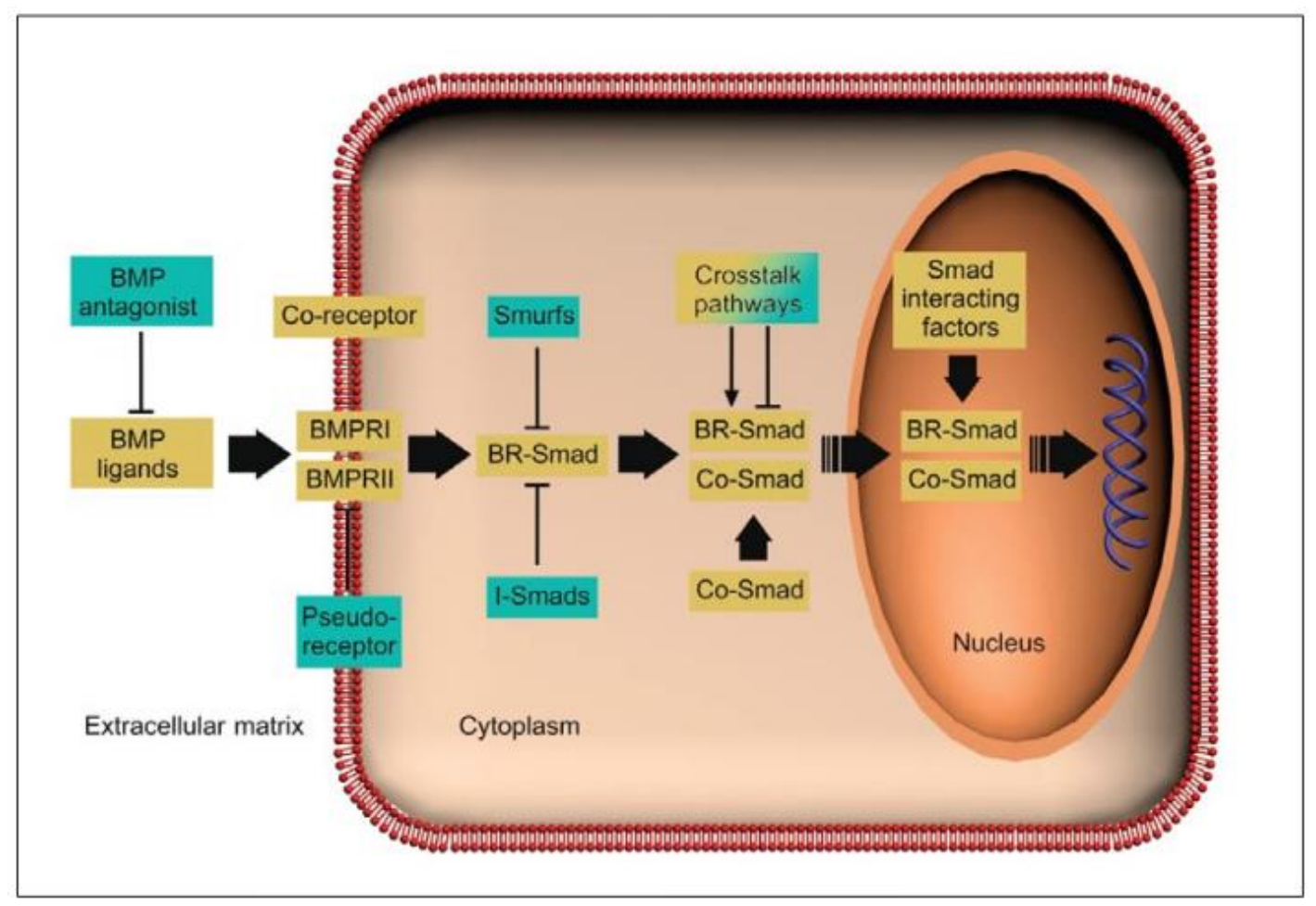

Fig. 1, Overview of BMP signalling. BMP signal flows from the extracellular environment to the nucleus. BMP ligands are secreted to the environment together with BMP antagonists. BMP ligands bind to BMP receptors (BMPRI and II) with the intervention of coreceptors. BMP antagonists avoid this binding and inhibit the signal transmission. Also, pseudoreceptors (equal to receptors but without signal-transmitting capacities) arrest the signal. The signal is transduced to BMP-specific intracellular effectors (BR-Smad). The transmission may be inhibited by binding of an inhibi-tory Smad (I-Smad) to BRSmad or BR-Smad can be tagged for destruction through Smurf-mediated ubiquitin binding. BR-Smad interacts with CoSmad and they form a complex which is translocated to the nucleus where, through its binding of a number of factors, it is able to generate the transcriptional response on target. Additionally, it may be produced interplay with other pathways at the level of BR-Smads

\section{The BMP extracellular world: the ligands and their antagonists}

BMPs are so called because they were originally discovered by inducing bone and cartilage formation. These proteins are the largest ligand subfamily in the TGF- $\beta$ super-family of cytokines dedicated to cellular signalling. BMPs also belong to the TGF- $\beta$ superfamily, the BMP-related ligand family of growth differentiation factors (GDFs), activins, inhibins, anti-Müllerian hormone and nodal. The BMP ligand family is composed of, among others, the following molecules: Bmp2, Bmp3, Bmp4, Bmp5, Bmp6, Bmp7, Bmp8a, Bmp8b, Gdf2 (also named Bmp9), Bmp10, Gdf11 (also named Bmp11), Gdf7 (also named Bmp12), Gdf5 (also named Bmp14), Gdf6 (also named Bmp13) and Bmp15. Bmp1, although named Bmp, is unrelated to the other Bmps as it is a 
protease involved in the cleavage of procollagen fibrils and chordin, a secreted antagonist of Bmp action [1].

All members of the TGF- $\beta$ superfamily are characterised by the presence of seven conserved cysteines involved in the tertiary and quaternary folding. The BMP family members can be distinguished from the TGF- $\beta$ and activin families by two extra conserved cysteines [2]. The active form of TGF- $\beta$ cytokines is a homodimer stabilised by hydrophobic interactions and strengthened in most cases by the formation of disulphide bridges between free cysteines conserved in each subunit. As an exception, Bmp15 and Gdf9 lack the cysteine to form the intersubunit disulfide bridge so that they may form both homodimers and heterodimers with each other. Also, activin, inhibin and other Bmp monomers can form heterodimers. For instance, heterodimers formed by Bmp2 and Bmp7 and Bmp4 and Bmp7 seem to have more potent activity than their corresponding homodimers [3]. Each subunit of Bmp ligand dis-plays several $\beta$-strands interlocked by three conserved dis-ulphide bonds that form the so-called "cysteine knot" [4]. Bmps are generated as large pre-proteins composed of a signal peptide, a predomain and a mature domain. After the secretion and elimination of signal peptide, the pre-proteins undergo dimerisation and, later, are cleaved by specific proteolytic enzymes to generate the active mature ligand.

Although Bmps were isolated initially as molecules secreted by skeletal cells, these proteins are synthesised in a wide number of cells where they play a key role in tissue homeostasis and in cell proliferation and differentiation control. However, the main role of Bmps, and whereby they are initially identified, is the regulation of bone generation through control of osteoblast differentiation from mesenchymal cells. Furthermore, Bmps can induce cartilage formation both through the stimulation of maturation of chondrocytes and through an increase in their activity. The Bmps with the greatest involvement in osteogenesis are Bmp2, Bmp4, Bmp5, Bmp6, Bmp7 and Bmp9. Bmp2is expressed in periosteal and osteogenic areas while Bmp4and Bmp7are expressed in perichondrium [5]. However, the transcriptional and posttranscriptional regulation of Bmp expression is not clear, although Bmp4auto-regula-tion is probable because of its Bmpdependent expression in skeletal cells [6]. Bmp2 and Bmp4 overexpression in developing limbs triggers an increase in the number of chondrocytes, which, in turn, enhances joint fusions. Also, Bmp2 and Bmp4 are expressed in trabecular bone in epi-physes of long bones, compact bone in diaphyses of long bones, cranial flat bones and jaw bone [7]. However, the functions of Bmp ligands go beyond their role in osteogenesis and a number of their functions are not related to bone generation and maintenance. For instance, mice mutants for Bmp2are lethal because they display defects in cardiac development and in the periembryonic tissues and Bmp4mutants are also not viable because they lack mesodermal differentiation. In addition, Bmp7null mutants do not form eyes and lack glomerular development, which leads to renal failure after birth. Also, disruption of Bmp8triggers infertility because of defects in spermatogenesis, since this Bmp is expressed in testicles. Also, Bmps are able to control different steps of differentiation and specification of several embryonic cell types to give rise to distinct components of the vertebrate nervous system [8]. Likewise, in embryonic development Bmps have a critical role in inducing apoptosis, a necessary process to remove cells and tissues that are already useless. Finally, Bmp2, Bmp4 and Bmp6 were located in areas of vascular calcification and it has been shown that Bmp2 is able to inhibit vascular smooth muscle cells when they are properly stimulated [5].

The regulation of BMP pathway activity may be carried out on several levels, the first of which is the co-secretion of antagonist molecules of Bmp ligands to the extracellular environment, called ligand traps by some authors (Fig. 2). These antagonists are able to attach to Bmp ligands, preventing them from binding to the correspondent receptors through blocking of binding sites (as has been shown for Bmp7 and its antagonist, noggin; see below). The binding of these antagonists displays different affinity depending on the Bmp ligand, so that the members of this group of proteins work in synergic and complementary forms rather than as redundant inhibitory signals. Most of these proteins were discovered in Xenopusembryos by their capacity to inhibit the ventralising effects of Bmps, inducing dorsalisation and neural tube formation. The structure of Bmp antagonists is similar to that of Bmps and displays con-served cysteine-rich domains (CR domains), which form the structure called the "cysteine knot". Depending on the location of the cysteine residues, the Bmp antagonists are classified in several groups: noggin, chordin family, 
twisted gastrulation and Dan family. Most of these antagonists are synthesised in osteoblasts together with Bmp ligands and their expression seems to be controlled by the same Bmps, which leads to a self-regulation circuit.

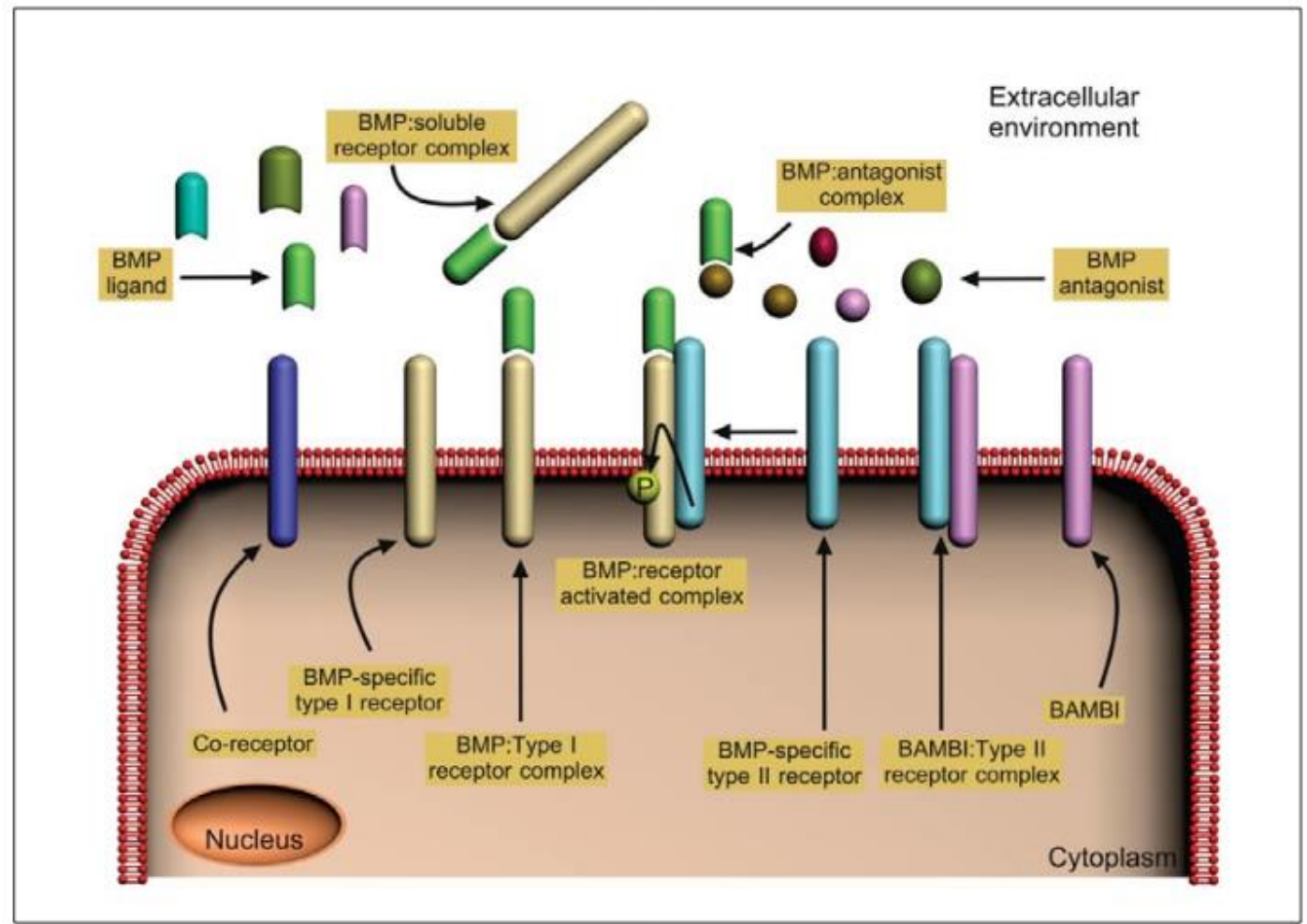

Fig. 2. BMP signalling start. In plasma membrane, there are two types (type I and type II) of serine/threonine kinase transmembrane receptors involved in signal transduction and each receptor has distinct versions with different capacities for binding different types of ligand molecules. The ligand-receptor interaction is facilitated by a number of transmembrane co-receptors. On the other hand, there are a number of soluble antagonist molecules that bind to BMP ligands, avoiding their binding to the receptors. Also, there are soluble forms of receptors which bind to ligands and interfere with the signal. The pathway begins when a member of the BMP ligand family binds to type I receptor and this preformed complex is able to bind to type II receptor and together they form the BMP:receptor activated complex. Type II receptor displays constitutive kinase activity so that when it is bound to type I receptor, this is phosphorylated and, thus, activated. Type I receptor is responsible for signal transduction into the cell. Also in the cell membrane there is decoy receptor molecule, named BAMBI, which is capable of binding to type II receptor but without transmitting a signal

Noggin is a 64-kDa glycosylated protein that, after its secretion, forms functionally active homodimers. The noggin structure displays, besides a CR region with the cysteine knot, a heparinbinding segment that retains the protein attached to the cell surface. Noggin is able to bind with distinct affinities to Bmp2, Bmp4, Bmp5, Bmp6, Bmp7, Gdf5 and Gdf6. The expression of noggin in osteoblasts is activated by Bmp2, Bmp4 and Bmp6 as a possible security system which avoids the overload of Bmp signal-ling into skeletal cells. A similar mechanism may exist in chondrocytes where noggin is upregulated by Indian hedgehog (IHH), another signalling molecule with a key role in the Hedgehog pathway, a pathway also involved both in development control and in differentiation/proliferation cellular balance. The in vitronoggin overexpression inhibits differentiation of mesenchymal cells in osteoblasts as well as arresting the osteoblast function when the cells are already differentiated. On the other hand, null mutants for noggin are lethal in mice, giving rise to severe errors in development such as failures in neural tube formation and skeleton [9]. 
Chordin is a $120-\mathrm{kDa}$ homodimer with four CR do-mains through which it interacts with Bmp2, Bmp4 and Bmp7. Like other Bmp antagonists, chordin displays a number of CR domains that are characterised by the lo-cation of ten cysteines that form the cysteine knot and by the presence of chordin typical motifs CXXCXC and CCXC. Chordin-like CR domains are present in a number of other extracellular secreted proteins involved in Bmp signalling such as Chordin like1/Ventroptin/Neuralin1, chordin like-2, procollagen type II, kielin, amnioless, nell, crossveinless2, crim-1 and members of the CCN family. Many of these proteins are also Bmp antagonists, but some can promote the Bmp action. Chordin expression is high in undifferentiated chondrocytes and its levels decrease in mature cartilage, whereas its expression in osteoblasts is low. The control of chordin function is carried out on the extracellular level through its interaction with a number of other secreted proteins. In this way, the zinc metallo-proteases Bmp1 (the only protein that, although named as Bmp and involved in Bmp signalling regulation, does not work as a cytokine but as an enzyme) and Tolloid, two proteins arising from an alternative splicing of a single gene, are of importance. Bmp1/Tolloid is able to bind to Chordin/Bmp complexes and cleave and separate chordin from them, destroying the biological properties of chordin and releasing fully functional Bmps. Therefore, Bmp1/Tolloid abolishes the antagonist effect of chordin on BMP signal-ling. The Bmp1/Tolloid activity is specific to Chordin/Bmp complexes and does not affect any another antagonist/Bmp complex. In addition, two proteases with similar activities to Bmp1/Tolloid have been depicted, tolloid like-1 and tolloid like-2, which contribute to the control of chordin function. In addition, on another level of regulation, the cleavage of chordin by Bmp1 is regulated by an additional Bmp antagonist, Tsg (twisted gastrulation). Tsg is able to bind to a complex formed by chordin, Bmp and Bmp1, enhancing Bmp1 action and favouring the release of Bmp from the complex. Tsg is a $23.5-\mathrm{kDa}$ secreted glycoprotein that dis-plays two conserved CR domains. The amino-terminal of these is similar to the CR domain of chordin and through this Tsg binds to Bmp2 and Bmp4 [9].

Another family of secreted glycoproteins capable of binding Bmps and working as antagonists of BMP signal-ling is Dan (differential screening-selected gene aberrative in neuroblastoma). This Dan family is composed of at least nine proteins: Gremlin, Sclerostin, Dan, USAG1 (uterine sensitisation associated gene 1), Cerberus, Caronte, Dante, and Protein related to Dan and Cerberus. All these proteins display a carboxy-terminal CR domain with nine conserved cysteines. Most of the members of this family are expressed during embryonic development and, except for gremlin and sclerostin, do not regulate BMP signal in adult skeleton. Gremlin is able to bind to Bmp2, Bmp4 and Bmp7 with high affinity. During embryonic development, gremlin is expressed in limb mesenchyme, lung and kidney. Consistent with this, gremlin null mutants in mice are lethal in early embryonic stages and display abnormalities in limb, kidney and lung airway development. After the birth, gremlin is expressed primarily in brain and gastrointestinal tissues. The importance of gremlin in bone formation is supported by the observation that in osteoblasts, gremlin overexpression leads to spontaneous fractures and osteopenia, because gremlin inhibits Bmp effects on osteoblast function and differentiation of marrow stromal cells. In addition, gremlin and other members of the Dan family such as cerberus, coco and sclerostin can also counteract the action of the Wnt signal (another important cell signal-ling mechanism that controls the proliferative impulse in tissue self-renewal), which suggests other mechanisms of work for this group of Bmp antagonists. In this way, sclerostin is able to bind to Wnt co-receptors and arrest the canonical pathway of $\mathrm{Wnt} / \mathrm{B}$-catenin. Additionally, sclerostin can bind to Bmp antagonist noggin and inhibit its negative effect on Bmp signalling. Besides, sclerostin may work as a Bmp antagonist and binds to Bmp2, Bmp4, Bmp6 and Bmp7, although with weak affinity. Sclerostin is a secreted glycoprotein specifically expressed in skeletal cells as chondrocytes, osteoblasts and osteocytes, and its expression is induced by Bmp2, Bmp4 and Bmp6 in osteogenic cell lines in culture. Dan is another glycoprotein secreted, of $19 \mathrm{kDa}$, which binds to Bmp2, Bmp4 and, also, Gdf5. Cerberus is a $31-\mathrm{kDa}$ secreted protein which in mice is expressed during embryonic development. Neither Dan nor cerberus null mutant display a conspicuous phenotype. Caronte, coco and dante seem to have importance during embryonic development but their expression and function are not clear [6]. In addition, we can include follistatin and FSRP follistatin-related protein as Bmp antagonists because although they bind to activin (another kind of TGF- $B$ ligand) with greater affinity, they are capable of binding to Bmp7, Bmp2, Bmp4 and Bmp6 with distinct specificities and affinities [9]. 


\section{BMP impact in cell membrane: the receptors and their antagonists}

All ligands of the TGF- $\beta$ superfamily bind to receptors that are transmembrane proteins with serine/threonine kinase activity. There are two types of receptors, named type I and type II receptors, and several types of coreceptors. Both types of receptors are formed by an aminoterminal extra-cellular domain, a transmembrane region and a carboxy-terminal intracellular serine/threonine kinase domain. The type I receptor contains in the amino-terminal area of intracellular kinase domain a characteristic sequence SGSGSG, named the GS domain, where the serines are susceptible to being phosphorylated by the type II receptor, which dis-plays constitutive serine/threonine kinase activity [4]. Only the phosphorylated type I receptor is active and displays kinase activity through which the signal is transduced. But for that type I receptor to be phosphorylated, proximity of type II receptor and the formation of a complex with the ligand, which acts as binding nexus, are necessary (Fig. 2).

For Bmp, the type I receptors are type I Bmp receptors Bmpr1a (also called Alk3 from activin like kinase-3) and Bmpr1b (or Alk6), and type I activin A receptor Acvr1 (or Alk2). Other type I receptors in humans are Alk5 and Alk1 for TGF-B ligands, Alk4 for activins and Alk7 for nodal. The type II receptors for Bmp are type II BMP receptor Bmpr2, only for Bmp ligands, and type II activin receptors Acvr2a (also named ActR-II) and Acvr2b (also named ActR-IIB), for activin and Bmp ligands. Other type II receptors in humans are TgfrII for TGF- 3 ligands and Amhr2 for the AMH ligand [10].

Furthermore, BMP signalling is enhanced by a number of coreceptors that work by capturing the ligand molecules and enhancing their binding to the type I and/or type II receptors (Fig. 2). Among these coreceptors is the proteoglycan betaglycan, sometimes named type III receptor. A recent study revealed that betaglycan, in addition to binding to TGF- $\beta$ and inhibin (its first identified ligands), is able to work as a Bmp cell surface receptor binding multiple Bmp-type ligands such as Bmp2, Bmp4, Bmp7 and Gdf5 [11]. Additionally, BMP signalling also is enhanced by other transmembrane coreceptors, named DRAGON and RGMa (repulsive guidance molecule a). Both DRAGON and RG-Ma are able to bind to Bmp2 and Bmp4 (but not to other ligands) and are also capable of interacting with the respective receptors Bmprla or Bmpr1b. Possibly, these coreceptors work interacting with ligands and their receptors at the same time, promoting thus their binding [3]. Finally, there is another betaglycan-related coreceptor, named endoglin, that functions by helping Bmp9 bind to its receptor [12].

The binding mechanism ligand/receptor is slightly different for Bmps than for TGF- $B$ and activin. In the case of Bmps, the ligands bind with more affinity to type I receptors and the preassembled type I receptor/ligand complexes display a higher affinity for type II receptors, which are recruited (Fig. 2) toward the preformed complexes [4]. In addition, it has been shown that BMPRII and type I receptors may form both homodimeric and heterodimeric complexes even before ligand signalling. Binding of ligands to these preformed complexes is able to trigger distinct responses depending on the nature of the complex. If the complex is heterodimeric (type I and type II receptors), the BMP canonical pathway through Smad intracellular messenger proteins is activated and if the complex is homodimeric the mitogen-activated protein kinases (MAPK) pathway is activated [2].

At this level, there is another regulating mechanism of BMP signalling: it involves pseudoreceptor BAMBI (Bmp and activin receptor membrane bound inhibitor, also called Nma), a transmembrane protein similar to type I receptors. BAMBI functions as a decoy receptor and competes with type I BMP genuine receptors for formation of ligand/receptor complexes, so that signal transduction is inhibited because BAMBI is unable to phosphorylate the intracellular messengers of the pathway and, therefore, it is unable to transmit the signal (Fig. 2). BAMBI is expressed in differentiated osteoblasts and during embryonic development plays a key role in embryo dorsalisation. Finally, BAMBI expression is upregulated by Bmps, TGF-ßs and Wnt signalling [4], thus generating a mechanism for autoregulation of BMP signalling and a point of connection between BMP signalling intensity and other pathways more or less functional and molecularly related to the BMP pathway, such as TGF- $B$ and Wnt pathways. 
There is an additional system of BMP signalling control. It involves soluble forms of receptors, secreted to the extracellular environment, which bind to ligands with the same intense affinity as transmembrane receptors (Fig. 2). Evidently, these soluble receptors are unable to transmit the signal into the cell because they lack a connection with the membrane and cytoplasm of the target cell. These soluble receptors compete thus with membrane-bound receptors by ligand capture and, therefore, they are capable of modulating BMP signalling. This is the case of Bmp4 signalling, which is inhibited by the soluble form of the type I receptor to which binds [3].

Finally, it has been demonstrated that endofin (endo-some-associated FYVE-domain protein) is capable of functioning as an anchor protein, establishing a bridge for interaction between the activated receptor complex and the BR-Smad Smad1 and, thus, facilitating Smad1 receptordependent phosphorylation and activation. Also, endofin contains a motif for binding to a phosphatase, which may negatively regulate BMP signalling through receptor de-phosphorylation [13].

\section{Sending BMP signals: the story of Smad's journey from the cytoplasm to the nucleus}

Both the transmission and modulation of signalling for Bmp and other ligands of the TGF- $\beta$ superfamily from the cell membrane to the nucleus are carried out in vertebrates by a group of eight intracellular proteins called Smads (Fig. 3). These proteins are classified depending on their function in three distinct groups. The R-Smads (receptor regulated Smads) are those that interact with the membrane receptors and are responsible for transducing the signal into the cell. The RSmad that interacts with the receptor is dis-tinct, depending on the ligand and receptor types that trigger the signal. In this way, Smad 2 and Smad3 only transmit the signal from TGF- $\beta$, Nodal or Activin ligands (and they are called Activin-regulated Smads or AR-Smads) and Smad1, Smad5 and Smad8 only transduce the signal from Bmp and Gdf ligands (and they are called Bmpregulated Smads or BR-Smads). But in endothelial cells TGF-ß ligand can activate Smad1, Smad5 and Smad8 through a tissue-specific type I receptor named ALK1. Another group of Smads are the Co-Smads, which work as Smad helpers, facilitating the achievement of R-Smad functions. In this group, the only integrant is Smad4, which is capable of interacting with any R-Smad. Finally, a third group of Smads are the I-Smads or inhibitory Smads, which are composed of Smad6 and Smad7. I-Smads' mission is to modulate the signalling, either competing with R-Smads by binding to receptors (acting as a Smad decoy) or through binding to R-Smads (Fig. 3) [14].

Both R-Smads and Co-Smads display two structurally conserved Mad homology (MH) domains, the amino-terminal MH1 domain and the carboxy-terminal MH2 domain, bound by a region of weak homology between Smads called as linker region. The R-Smads, but not the CoSmads, display in their carboxy-terminal end an SM/VS motif through which R-Smads' activation is achieved by phosphorylation of their serines by serin/threonin kinase activity of activated type I receptor. The MH1 domain of Co-Smad and most of the R-Smads, except for one Smad2 isoform, is able to bind directly to DNA or interact with other cofactors that are capable of binding to DNA, as well as containing the nuclear localisation signal, which plays an important role in translocation to the nucleus of activated Smads.

In addition, the MH1 domain keeps the MH2 domain switched off by binding to it when the Smad is inactive. The MH2 domain is responsible for interaction with the receptor and it contains in its carboxy-terminal end, as has been previously explained, a motif with serines, which are a target for phosphorylation by the activated receptor that gives rise to Smad activation. On the other hand, the MH2 domain is highly conserved between all Smad proteins and this allows Smads to interact with other Smad proteins for the formation of homo- and heteromeric complexes that are important for both achievement of Smad signalling function and its regulation through I-Smads. In addition, the $\mathrm{MH} 2$ domain has direct contact with proteins that make up the nuclear pore and this makes the Smad shuttling from cytoplasm to nucleus possible. The MH2 domain is also capable of binding to other DNA binding factors and it can also bind to coactivators and corepressors for triggering the transcriptional response to the signal in target genes. Finally, the linker region, although poorly conserved between Smads, displays a number of motifs and residues that are 
utilised for interaction with other pathways as well as for Smad tagging for its elimination and turnover [4], as will be depicted below.

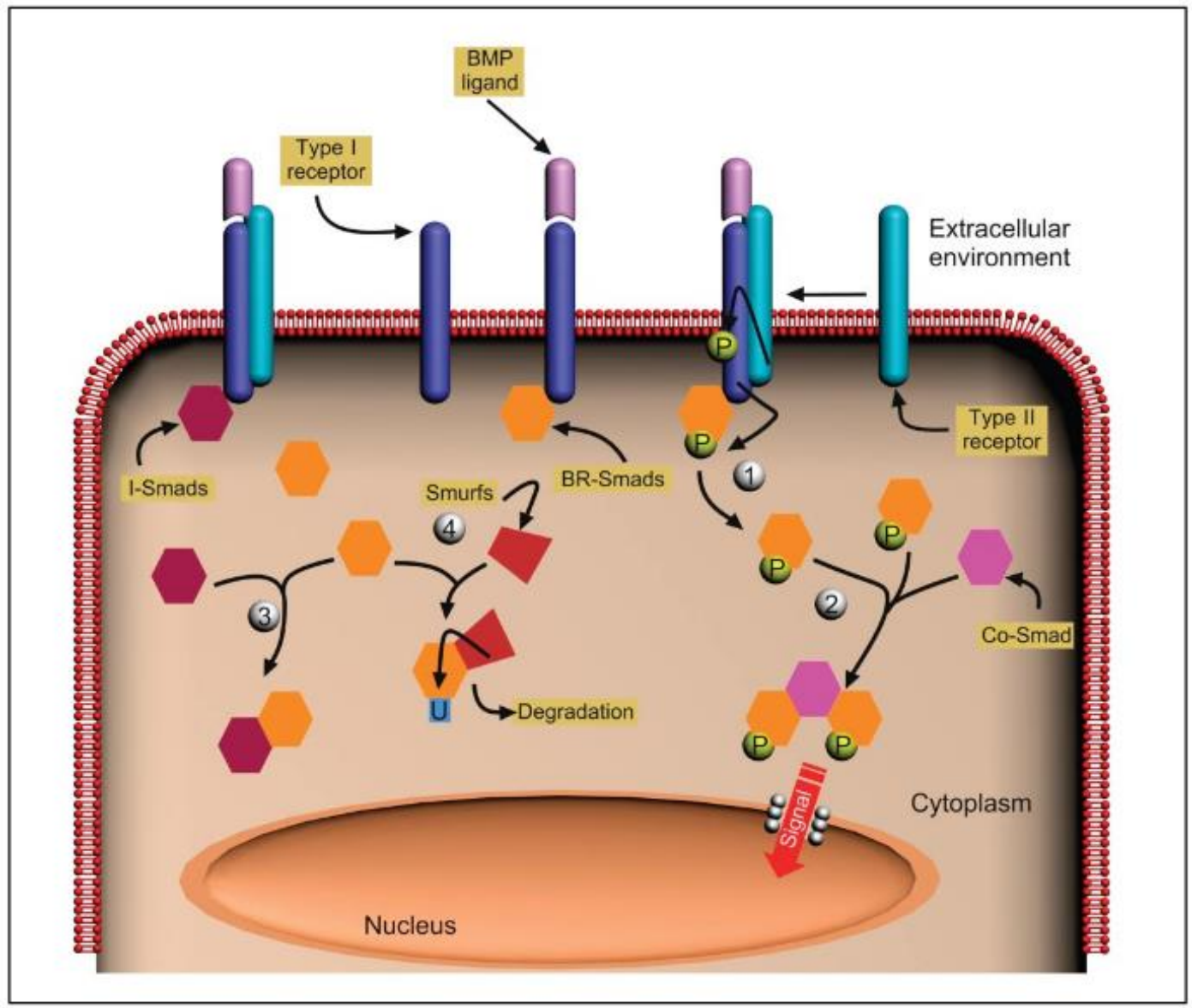

Fig. 3. Intracellular behaviour of Smads in BMP signalling. (1) The type I receptor activated (after phosphorylation by type II receptor) phosphorylates a BR-Smad and, thus, sends the signal into the cell. (2) These activated BR-Smads, after release of the receptor, form heteromeric complexes with Co-Smad, and together cross to the nucleus to develop their function. At this level there are two main regulation mechanisms. One is based (3) on the inhibitory effect of I-Smads on BR-Smads because the former bind and immobilise the latter and this prevents the inter-action with the activated receptors; in addition, I-Smads bind to receptors, avoiding the binding of BR-Smads. (4) Another regulatory mechanism is based in BR-Smad ubiquitination by Smurfs, which leads to their proteasomal degradation

When the cell is in a non-stimulated state, the R-Smads are principally located in the cytoplasm, while the I-Smads are principally in the nucleus and Smad4 is uniformly distributed throughout the entire cell. When the signal reaches the cell, the type I receptor is phosphorylated by the type II receptor in the GS region, which promotes a conformational change that triggers its activation. The activated type I receptor is now capable of phosphorylating the serines into the RSmad SM/VS target motif. It is probable that the R-Smad SM/VS motif, once phosphorylated, competes with the GS region of the receptor by binding to the specific site for interaction in the MH2 domain of R-Smad. Thus, the phosphorylated R-Smads lose affinity to the receptor and are released into the cytoplasm in their active form. Later, the R-Smads are translocated to the nucleus, a process depending on previous phosphorylation, so that only when R-Smads are activated they tend to accumulate in the nucleus [4]. 
The R-Smads, once phosphorylated, are able to form heteromeric complexes with Smad4 (Fig. 3 ). These complexes can be constituted by one activated R-Smad bound to Smad4 as well as by two activated R-Smads bound to Smad4, depending on the target gene and depending on other recruited factors to the complex once it is shuttled to the nucleus [15].

For the shuttling of R-Smads into the nucleus, at least for Smad1 and Smad3, a nuclear location signal which comprises the lysine-rich motif KKLKK (located in the MH1 domain), which is conserved between all the R-Smads [4], has been suggested. However, although upon ligand stimulation R-Smads tend to accumulate in the nucleus, R-Smads and Smad4 do not remain permanently in the nucleus but are constantly shuttled between the nucleus and cytoplasm, even in the presence of a signal. This requires the existence of a phosphorylation/dephosphorylation cycle, carried out respectively by the kinase activity of the activated type I receptor and by phosphatases located in the nucleus [14].

Once in the nucleus, the complexes formed by R-Smads and Smad4 bind to DNA either directly or through interaction with a universe of transcription factors to exercise the effects of activation and repression on target gene expression. One of the Bmp-regulated Smads, Smad1, is able to bind to DNA through GC-rich sequences GCCGNC or GRCGNC, located in the promoters of some of its target genes such as Smad6(an I-Smad) or Id-1 (inhibitor of differentiation). These sequences are normally accompanied by AGAG or GRCT motifs, through which Smad4 interacts with DNA, reinforcing the intensity of DNA binding by the complex. However, the capacity of Smads for binding to DNA is weak and the participation of other DNA binding factors (which are recruited by the same Smads) is necessary to achieve a greater affinity and also more specificity.

The transcription factors, which are recruited by R-Smads into the nucleus, have a diverse nature and fulfil distinct roles, activating or repressing distinct target genes in a specific manner. In the case of Bmp-regulated Smads, few partners for interaction with DNA have been characterised. One of the best studied is the Schnurri factor of Drosophila, which acts as a cofactor of BR-Smads and Co-Smad homologues in the activation/repression of target genes. Another transcription factor important for the function of R-Smads is polyomavirus enhancer-binding protein 2 (Pebp2), which is composed of two subunits, of which the subunit $\beta$ is variable and can be one of the proteins Runx, Runx1, 2 or 3. Any of these three types of subunits $\beta$ can interact with Smads, both Bmpand TGF- $\beta$ regulated. However, Runx 2 seems to be the most important for Bmp-mediated signalling since the interaction between Runx2 and the Bmp-dependent Smad1 has been demonstrated in some promoters of target genes. Furthermore, Runx2 seems important for induction of differentiation in osteo-blasts [14].

Another nuclear cofactor that interacts with all TGF- $B$ and Bmp-regulated Smads is Ski (oncoprotein homologue to the transforming protein (v-Ski) of avian Sloan Kettering retrovirus), which fulfils a signal inhibitory mission. Ski is able to inhibit the signal through three mechanisms: first, through the stabilisation of R-Smad:DNA complexes, avoiding the turnover with newly activated R-Smads; second, by interfering with the binding of R-Smads to nuclear cofactors; and third, by attracting corepressors and histone deactylases to R-Smad:DNA complexes. The key role of Ski in development has been demonstrated in mice where null mutants for Ski show muscular and skeletal abnormalities and Ski overexpression causes osteopenia.

In addition, another protein that is involved in BMP signalling modulation, through its inhibitory effects, is Tob (transducer of Erbb2), which is also involved in the control of processes of proliferation and cell differentiation. Tob is capable of binding to Bmp-specific type I receptors, simulating and competing with Bmp-regulated Smads. Also, Tob can bind to these R-Smads, avoiding their binding with the Co-Smad Smad4 (in a similar function to that carried out by ISmads, as will be depicted below). On the other hand, Tob is upregulated by BMP2, which makes evident the existence of a negative feedback cycle for the control of BMP signalling. Finally, Tob inactivation gives rise to an increase in bone formation through an increase in the number of osteoblasts by enhancing their differentiation [6].

In addition, Smad1 may also bind to Hox proteins (that contain homeobox domains), essential for development and, in this case, osteoblast differentiation and bone generation [2]. 
On the other hand, Smad6 and Smad7, the inhibitory Smads or I-Smads, are able to bind to type I BMP recep-tors so as to avoid the signal transmission, since they work as decoy R-Smads. Besides competing with R-Smads in binding to the receptors, Smad6 is capable of competing with Smad4 in binding to activated BR-Smad Smad1, so that Smad6:Smad1 complexes are formed that are unable to lead to some response (Fig. 3). Thus the Bmp signal intensity is modulated because the number of Smad4:Smad1 active complexes is reduced. Smad7, like Smad6, is also able to bind to Bmp receptors in competition with BR-Smads, but it is a less selective inhibitor since it is also capable of binding to TGF- $\beta$-specific receptors. On the other hand, Bmps may control Smad6 expression since in its promoter there are a number of binding motifs for Bmp-specific R-Smads Smad1 and Smad5, so that the existence of a mechanism of autoregulation is possible. Additionally, Smad7 expression is also under the control of BMP signal-ling [6, 15].

\section{Understanding unsuspected collateral effects of BMP signalling: from canonical pathway to crosstalk with other pathways}

Like the Smads are phosphorylated in their carboxy-terminal end in a ligand-dependent and specific manner by membrane receptors, R-Smads and Smad4 can also be phosphorylated through kinases involved in other path-ways, such as MAPKs Erk1 and Erk2 (extracellular signal regulated kinases), Jnk (Jun N-terminal kinase) and p38. This is the case for Smad1, which is phosphorylated in its linker region through kinases of the MAPK pathway. This phosphorylation is independent of its phosphorylation and activation carried out by membrane receptors through the BMP canonical pathway. The Smad1 phosphorylation through MAP kinases leads to the repression of transcriptional activity (and therefore to the inhibition of Bmp-mediated responses) through mechanisms such as the inhibition of nuclear transport or facilitating the Smurf1 binding to Smad1, which gives rise to Smad1 ubiquitination and posterior degradation. Indeed, activators of the MAPK pathway inhibit the Bmp effects in several development processes such as lung morphogenesis, generation of the nervous system and limb formation [14].

Conversely, the BMP pathway may induce the activation of MAPKs, JNK and p38. This activation leads to the phosphorylation and consequent inhibition of the function of a cytoplasmatic protein called associated molecule with the SH3 domain (AMSH). This protein, in its active state, is able to interact with the I-Smad Smad6 and avoid its binding to type I BMP receptors and, therefore, enhancing Bmp-mediated signal transmission. When the Bmp-dependent activation of JNK and p38 pathways occurs, the phosphorylation and inactivation of AMSH is triggered, which, in turn, causes the arrest of inhibitory action of AMSH on Smad6. Thus, Smad6 can already bind to type I BMP receptors and inhibit BMP-mediated signal transmission. Thus, a negative self-regulation cycle on BMP signalling is achieved [6].

In addition, a connection between the BMP pathway and microRNA-mediated gene regulation has recently been established. MicroRNAs (miRNAs) are small RNA molecules capable of repressing gene expression, interfering with RNA translation or affecting RNA stability. In this way, it has been depicted that Smads are able to control miR-21 processing and, therefore, its effects. This finding is of great importance since miR-21 is one of the miRNAs that is deregulated in almost all tumours, and they confer invasive and metastatic ability, as occurs in colorectal cancer. Another function of miR-21 is triggering PDCD4 repression, a transcription factor that controls the vascular smooth muscle cell phenotype capable of turning on the differentiation programme from somatic stem cells to vascular smooth muscle cells. Smads intervene in miR-21 maturation through their interaction with DEAD box RNA helicase p68, which forms part of the Drosha microprocessor complex, an RNase responsible for the first cleavage of primary miRNAs (primiRNA) to render pre-cursor miRNAs (pre-miRNAs) in the nucleus. Concretely, p68 interacts with Smad1, Smad5 (BR-Smads) and Smad3 (AR-Smad). However, it has not been possible to establish an interaction of p68 with Smad4 or with I-Smad Smad6. On the other hand, after BMP stimulation, interaction of Smad1 and Smad5 with Drosha complex is also produced and, unlike the association of Smad1 and Smad5 with p68, this interaction is independent of primiRNA presence in the Drosha complex. The interaction Smads:p68 is able to increase the amount of mature miR-21 through its post-transcriptional regulation, accelerating or facilitating the conversion of pri-miR-21 to pre-miR-21. So far, it is unknown if there are other miRNAs whose 
maturation is controlled by interaction between Smads, p68 and/or Drosha, and the exact mechanism by which the interaction of Smads with microprocessor complex is able to enhance miRNA maturation is also unknown [16].

\section{Dismantling the BMP mechanism: Smurfs and more}

Another way Smads may be regulated is through ubiquitin-mediated degradation or modification. Ubiquitin is a small polypeptide (76 amino acids) that is covalently bound to lysine residues in target proteins. The ubiquitin binding to the target proteins involves a complex of three enzymes that work sequentially. Firstly, the E1- or ubiquitin-activated enzyme acts, catalysing the adenylation of ubiquitin in its carboxy-terminal end. Later, the E2- or ubiquitin-conjugating enzyme acts, transferring the ubiquitin from E1 to the specific site of the target protein. Finally, the ubiquitin is bound covalently to the target protein with the collaboration of ubiquitin ligase or enzyme E3, which is al-so responsible for the reaction specificity. The most important role for the ubiquitin is tagging the proteins for their degradation through the proteasome complex. Normally the signal for the degradation of a given protein is com-posed of a poly-ubiquitin chain, which is achieved because ubiquitin displays lysine residues, to which other ubiquitin molecules may also bind. However, the addition of a distinct number of ubiquitin molecules to proteins instead of leading to protein degradation may give rise to a number of other responses such as changes in subcellular localisation or structural and activity modifications [6].

The ubiquitination of R-Smads is carried out by a number of E3 ubiquitin ligases, among which are the SMURFs (Smad ubiquitination regulatory factors) Smurf1 and Smurf2. Smurf1 is capable of tagging with ubiquitin to BMP-specific receptors and BR-Smads Smad1 and Smad5 so that their degradation is enhanced. In addition, Smurf1 is able to enhance the interaction between I-Smads and type I receptors so that BMP-mediated signalling is inhibited [6]. On the other hand, Smurf2 can interact with both Smad1 and Smad2.

Besides interacting with the R-Smads, negatively regulating the signalling via BMP/TGF- $B$, Smurfs can also interact with I-Smads so that, through their degradation, they are able to act positively on the regulation of the pathway. Smurf2 interacts with Smad7 and can enhance its ubiquitination and mediate its degradation at a low level. In addition, Smad7 can enhance the Smurf2-mediated ubiquitination through stabilisation of the protein complexes of enzymes responsible for ubiquitination.

Another E3-ligase called Arkadia also interacts with I-Smads. Arkadia binds to Smad7 and facilitates its poly-ubiquitination and degradation. Two other Smurf-related proteins, NEDD4-2 (neuronal precursor cell expressed, developmentally downregulated 4-2) and WWP1/TiuL1 (WW domain containing E3 ubiquitin ligase/TGIF-interacting ubiquitin ligase 1), are also capable of enhancing R-Smad degradation and negatively regulate the signalling via BMP and TGF- $\beta$ [14].

On the other hand, the Smurfs and Smurf-related proteins are not able to interact with Smad4, as this lacks the specific site for ubiquitination by the Smurfs and Smurf-related proteins. Smurfs and Smurf-related proteins can however achieve the degradation of Smad4 through an indirect mechanism when the R-Smads or I-Smads (which are capable of binding to Smurfs and Smurfrelated proteins) interact with Smad4. In addition, there are E3 ubiquitin ligases such as Ecto/TIF1 $\gamma$ (a component of the SCF E3 ligase complex and ectodermin), which interact specifically with Smad4, enhancing the Smad4 ubiquitination and degradation. However, Smad4 is relatively stable and it is rare for it to be poly-ubiquitinated and tagged for degradation. Instead, Smad4 is mono- or oligo-ubiquitinated, giving rise to other responses. For instance, the Smad4 mono-ubiquitination facilitates the formation of complexes between Smad4 and the activated R-Smads so that the signal is enhanced. 
Additionally, Smad4 can be modified by SUMO (small ubiquitin-like modifier), a 98aminoacid protein, similar to ubiquitin, which binds to lysine residues of proteins it modifies. The binding of SUMO to Smad4 may cause changes in Smad4 transcriptional activity. Given that Smad4 is involved in BMP signal transmission, the Smad4 modification by SUMO could elicit important effects on BMP signalling.

Another modification to which Smads are subjected is acetylation. For instance, Smad7 may be acetylated by the coactivator $\mathrm{p} 300$. Smad7 acetylation prevents the formation of the Smurf:Smad7 complexes so that Smad7 is protected from Smurf-mediated degradation. Thus, Smad7 can amplify its inhibitory activity on the BMP/TGF- $\beta$ signalling. Inversely, Smad7 may also be deacetylated by histone deacetylases and, thus, its ubiquitination and degradation are increased [14].

Finally, phosphatase-mediated dephosphorylation is another mechanism by which Smads are not degraded but deprived of their function. As has been previously depicted, the activated Smads are not permanently phosphorylated but their phosphorylation is dynamic. Thus, Smads are dephosphorylated in the nucleus to return to be activated in the cytoplasm by membrane receptors. Among the phosphatases involved in this process are the pyruvate dehydrogenase phosphatase (PDP) and the RNA polymerase II small C-terminal phosphatases (SCPs), which have been involved in the dephosphorylation and deactivation of Smad1. The SCPs can also eliminate the phosphorylation in the Smad1 linker region, modulating its transcriptional activity and its interaction with other pathways. In addition, magnesium-dependent PPM1A phosphatase dephosphorylates and disables the entire set of BR-Smads (Smad1, Smad5 and Smad8) and ARSmads (Smad2 and Smad3).

All these events give rise to a fine-tuning of Smad regulation and activity through a combination of the effects of kinases with the effects of phosphatases [14].

\section{BMPs in stem cells}

BMP proteins regulate fundamental biologic processes during embryonic and postnatal development through their involvement in the maintenance of an undifferentiated state in adult and embryonic stem cells as well as in the differentiation of specific lineages of certain stem cells and in cell fate decisions. BMPs not only induce the formation of cartilage and bone, but also play an important role in a number of non-osteogenic developmental processes [17]. For instance, it has been demonstrated that the BMP7 and BMP4 signal coming from mesenchyme plays a key role in branching morphogenesis during prostate development. Thus, the action of these BMPs on prostatic proliferative epithelium prevents an uncontrolled growth, preventing a random expansion and instead causing it to form branched structures that constitute the normal morphology of prostate $[18,19]$.

BMPs usually inhibit cell growth with the exception of some systems. It has been shown that BMP receptor IA directly inhibits proliferation in the stem cell niches of the intestine and the skin and indirectly regulates haematopoietic stem cells. Conditional inactivation of BMP receptor IA in mice leads to the expansion of stem cell populations in hair follicle, intestine and bone marrow, resulting in tumours in hair follicles, intestinal polyposis, and abnormal bone growth and expansion of haematopoietic stem cell population (reviewed in [20]). It has been established that in the skin and in the intestine, coordination between the growth-promoting effect of Wnt signalling and the transient expression of the Bmp antagonist Noggin is required to overcome the antigrowth effect of Bmp and activate skin and intestine stem cells [20]. If this Wnt-Noggin-BMP signalling balance is disrupted, intestinal and skin stem cells may proliferate without restriction to become cancer stem cells (CSCs). Therefore, loss of BMP signalling and/or abnormal activation of the Wnt cascade would lead to tumours in the skin and in the intestine. 


\section{BMPs in cancer}

The BMPs also have shown a critical role in epithelial-stromal interaction in the microenvironment of bone metastasis [20]. Bone provides chemotactic factors, adhesion factors and growth factors that allow carcinoma cells to target the skeleton and proliferate there. BMP target genes encode for osteoblast proteins and for osteoblast-specific transcription factors. Prostate cancer is frequently accompanied by osteosclerotic bone metastasis. Many studies have shown that BMP expression increases in the progression of prostate cancer (reviewed in [20]). Preclinical data suggest that prostate cancer cells promote osteoblastic activity through BMP6. In addition BMPs confer to prostate cancer cells the ability to invade the bone microenvironment [20]. Accordingly, recombinant Noggin, which inhibits the function of BMPs, could constitute a new possible therapeutic strategy for painful osteosclerotic bone metastasis in prostate can-cer [21]. Furthermore, in metastatic prostate cancer, BMPs, in particular BMP7, promote VEGF expression, and VEGF contributes to the osteoblastic metastatic lesions [21].

Furthermore, several findings suggest the involvement of BMPs in colon cancer and breast cancer.

BMPs have been shown to have an impact on the behaviour of breast cancer. The analysis of the expression of BMPs in breast carcinomas has demonstrated a differential expression pattern and the potential prognostic value of BMP2 and BMP7 for the patients [22]. BMP2and BMP7 had a contrast pattern of expression in normal and tumour tissues. The BMP2transcript level was lower and the BMP7transcript level was higher in breast tumours than in normal tissues. BMP2transcript was also significantly lower in tumours from patients with a moderate and poor prognosis than in those from patients with a good prognosis $(p=0.04)$. Also both BMP2 and BMP7showed a significant difference between node-positive and node-negative tumours $(\mathrm{p}=0.033$ and $\mathrm{p}=0.031$ respectively) [22].

Recent findings suggest that the BMP pathway may be inactivated in the majority of sporadic colorectal cancers [23]. Furthermore, it has been shown that up to $50 \%$ of individuals with juvenile polyposis, and inherited syndromes with a high risk of developing colorectal cancer, carry germline mutations in BMPR1A(bone morphogenetic protein receptor, type IA) or SMAD4genes [24, 25]. Additionally, patients with wild-type SMAD4have a three-fold higher benefit of 5fluorouracil-based adjuvant chemotherapy than patients with mutated SMAD4[26].BMP2 may act as a tumour suppressor gene, blocking cell growth and promoting apoptosis in mature colonic epithelial cells, in fact BMP2 expression is lost in adenomas of patients with familial adenomatous polyposis [27].

\section{Conclusions}

CSCs are a cellular subtype in tumours, with stem cell-like features. It is thought that these CSCs play a similar role to that of stem cells in normal tissues so that they are responsible for the growth and maintenance of tumours since they can generate the remaining cells of tumours (Fig. 4). These cells, which are the main components of tumour, display properties similar to normal differentiated cells, without the capacity for division and, therefore, they are unable to regenerate the tumour once eliminated by conventional drugs and/or surgical procedures. CSCs may arise from any type of normal cell in tissue: stem cells, progenitor (transit-amplifying) cells or even differentiated cells. These normal cells may undergo alterations in key genes that keep the proliferative activity of cells under control. These genes are involved in signalling pathways responsible for the proliferative control and differentiation during embryonic development and for the maintenance of tissue homeostasis and regeneration in adulthood. These signalling pathways are, among others, Wnt, TGF- $B$, BMP, Notch and Hedgehog. BMPs are a subfamily of ligands belonging to the super-family of TGF- $\beta$ cytokines involved in the maintenance of a quiescence state of stem cells in their niche and, later, in their differentiation to mature cells in normal tissues. Thus, alteration in components of the BMP signalling pathway may lead to uncontrolled proliferative activity of normal stem cells, breaking the fine balance between quiescence and 
proliferation. Likewise, alteration in BMP pathway components may lead to accumulation of cells without a fully mature phenotype due to failure in the signal that drives differentiation. Thus, it is clear that alterations in the BMP pathway may be an important milestone in the genetic and molecular events that lead to cancer development. In this way, it has been shown that alterations in distinct components of the BMP pathway are present in several tumour types such as prostate, colon and breast cancer. So, components of the BMP pathway are undoubtedly among the targets in the design of future drugs removing CSCs in tumour treatment.

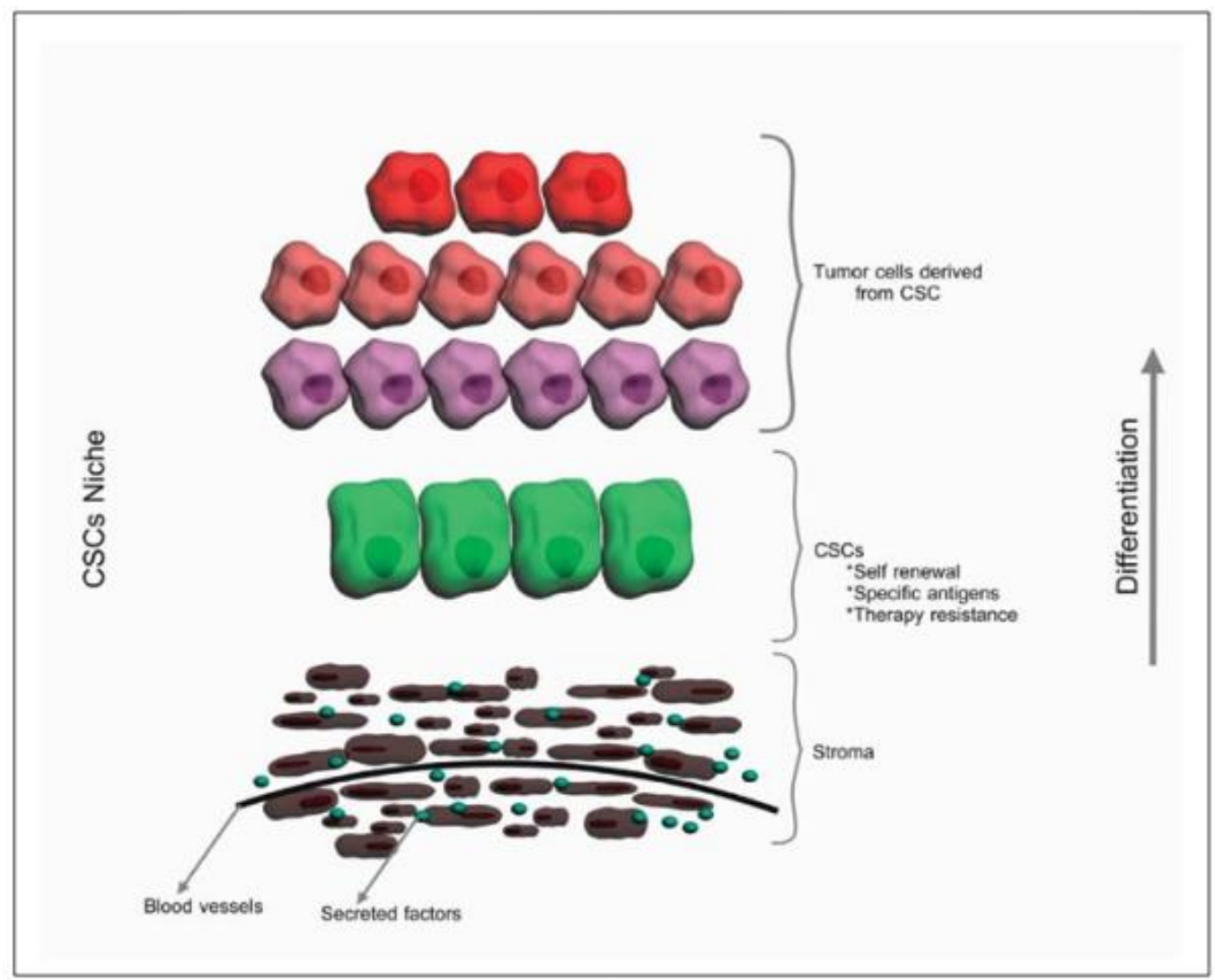

Fig. 4. Model of CSCs in solid tumours. Each solid tumour contains a "CSC niche", placed near the fibroblasts and the blood vessels of the tumour stroma. The "CSC niche" secretes factors that allow autocrine and paracrine CSC self-renewal. Furthermore, CSCs give rise to non-self-renewing tumour cells that constitute the bulk of the tumour. Additionally, CSCs may express specific antigens and may also be drug resistant. Adapted from [26]

Conflict of interest. Enrique Grande and Miriam Bolos are employed by, and own stock in, Pfizer. They were involved in the conception and writing, and approval of the final manuscript.

Acknowledgements. MBC is supported by Instituto de Salud Carlos III (Spain Goverment) through its research support programme. SDP has a contract with the Isidro Parga Pondal programme from Xunta de Galicia (Spain). VMV and GAG are supported by A Coruña Uni-versity Hospital Foundation. 


\section{References}

1. Canalis E, Economides AN, Gazzerro E (2003) Bone morphogenetic proteins, their antagonists, and the skeleton. Endocr Rev 24:218-235

2. Wan M, Cao X (2005) BMP signaling in skeletal development. Biochem Biophys Res Commun 328:651657

3. Lin SJ, Lerch TF, Cook RW et al (2006) The structural basis of TGF- $\beta$, bone morphogenetic protein, and activin ligand binding. Reproduction 132:179-190

4. Shi Y, Massagué J (2003) Mechanisms of TGF-beta signaling from cell membrane to the nucleus. Cell 113:685-700

5. Xiao YT, Xiang LX, Shao JZ (2007) Bone morphogenetic protein. Biochem Biophys Res Commun 362:550-553

6. Gazzerro E, Canalis E (2006) Bone morphogenetic proteins and their antagonists. Rev Endocr Metab Disord 7:51-65

7. Kochanowska I, Chaberek S, Wojtowicz A et al (2007) Expression of genes for bone morphogenetic proteins BMP-2, BMP-4 and BMP-6 in various parts of the human skeleton. BMC Musculoskelet Disord 8:128-137

8. Varga AC, Wrana JL (2005) The disparate role of BMP in stem cell biology. Oncogene 24:5713-5721

9. Balemans W, Van Hul W (2002) Extracellular regulation of BMP signaling in vertebrates: a cocktail of modulators. Dev Biol 250:231-250

10. Mazerbourg S, Sangkuhl K, Luo CW et al (2005) Identification of receptors and signaling pathways for orphan bone morphogenetic protein/growth differentiation factor ligands based on genomic analyses. J Biol Chem 280:32122-32132

11. Kirkbride KC, Townsend TA, Bruinsma MW et al (2008) Bone morphogenetic proteins signal through the transforming growth factor-beta type III receptor. J Biol Chem 283:7628-7637

12. David L, Mallet C, Mazerbourg S et al (2007) Identification of BMP9 and BMP10 as functional activators of the orphan activin receptor-like kinase 1 (ALK1) in endothelial cells. Blood 109:1953-1961

13. Shi W, Chang C, Nie S et al (2007) Endofin acts as a Smad anchor for receptor activation in BMP signaling. J Cell Sci 120:1216-1224

14. Ross S, Hill CS (2008) How the Smads regulate transcription. Int J Biochem Cell Biol 40:383-408

15. Massagué J, Seoane J, Wotton D (2005) Smad transcription factors. Genes Dev 19:2783-2810

16. Davis BN, Hilyard AC, Lagna G, Hata A (2008) SMAD proteins control DROSHA-mediated mi-croRNA maturation. Nature 454:56-61

17. Hogan BL (1996) Bone morphogenetic proteins in development. Curr Opin Genet Dev 6:432-438

18. Lamm ML, Podlasek CA, Barnett DH et al (2001) Mesenchymal factor bone morphogenetic protein 4 restricts ductal budding and branching morphogenesis in the developing prostate. Dev Biol 232:301-314

19. Grishina IB, Kim SY, Ferrara C et al (2005) BMP7 inhibits branching morphogenesis in the prostate gland and interferes with Notch signaling. Dev Biol 288:334-347

20. Ye L, Lewis-Russell JM, Kyanaston HG, Jiang WG (2007) Bone morphogenetic proteins and their receptor signaling in prostate cancer. Histol Histopathol 22:1129-1147

21. Dai J, Kitagawa Y, Zhang J et al (2004) Vascu-lar endothelial growth factor contributes to the prostate cancer-induced osteoblast differentiation mediated by bone morphogenetic protein. Cancer Res 64:994999

22. Davies SR, Watkins G, Douglas-Jones A et al (2007) Bone morphogenic proteins 1 to 7 in human breast carcinoma. J Clin Oncol, 2007 ASCO Annual Meeting Proceedings 25[18S], Abstract 21154

23. Kodach LL, Wiercinska E, de Miranda NF et al (2008) The bone morphogenetic protein pathway is inactivated in the majority of sporadic colorectal cancers. Gastroenterology 134:1332-1341

24. Howe JR, Bair JL, Sayed MG et al (2001) Germline mutations of the gene encoding bone morphogenetic protein receptor $1 \mathrm{~A}$ in juvenile polyposis. Nat Genet 28:184-187

25. Haramis AP, Begthel H, van den Born M et al (2004) De novo crypt formation and juvenile polyposis on BMP Inhibition in mouse intestine. Science 303:1684-168

26. Boulay JL, Mild G, Lowy A et al (2002) SMAD4 is a predictive marker for 5-fluorouracil-based chemotherapy in patients with colorectal cancer. Br J Cancer 87:630-634

27. Hardwick JC, Van Den Brink GR, Bleuming SA et al (2004) Bone morphogenetic protein 2 is ex-pressed by, and acts upon, mature epithelial cells in the colon. Gastroenterology 126:111-121 